\title{
Morphometric Characterization of Marginal Zone Lymphoma
}

\author{
${ }^{1}$ Emergency Clinical County Hospital "Sf. Apostol Andrei” Constanţa \\ ${ }^{2}$ Faculty of medicine, University "Ovidius" Constanţa
}

\begin{abstract}
The morphometry in histopathology is used to characterize cell populations belonging to different tissues and to identify differences in their parameters with prognostic implications. To achieve morphometric examination were selected 6 of 8 cases identified as marginal zone lymphoma. For each case analysis was done on five fields, for each field measuring the parameters of 20 cells. The studied parameters were for cytoplasm: cytoplasmic area, maximum and minimum cytoplasmic diameter, cytoplasmic perimeter; for nucleus were measured: nuclear area, minimum and maximum nuclear diameter, nuclear perimeter, nuclear contour index, nuclear ellipticity index, nuclear irregularity index. Also the nucleo-cytoplasmic ratio was calculated in all studied cases.

Marginal zone lymphoma is characterized in terms of morphometric parameters by small cytoplasmic and nuclear area, and small nucleo-cytoplasmatic ratio of about $1: 1$. Nuclear contour index is small, accompanied by a large ellipticity index and an small index of nuclear irregularity. Standard deviations for measured morphometric parameters are variable, having high values for cytoplasmic and nuclear area, highlighting
\end{abstract}

Mariana Aschie

Professor, MD, PhD, Department of Pathology, Faculty of Medicine, "University "Ovidius" Constanța

Clinical Service of Pathology, Emergency County Hospital

145 Tomis Avenue, 900591 Constanţa, Romania

$+40745043505$

aschiemariana@yahoo.com the polymorphic nature of the cells. Morphometric aspects, with corresponding microscopic aspects of large and small lymphocyte proliferation with cleaved and uncleaved nuclei, fit this form of lymphoma in terms of clinical outcome in indolent lymphomas category.

Keywords: lymphoma, morphometry, histopathology

\section{Introduction}

The morphometry in histopathology field is used to characterize cell populations belonging to different tissues and to identify differences in their parameters with prognostic implications. The morphometry may be used in the development of automated diagnostic programs.

Non-Hodgkin lymphomas are a heterogeneous group so morphological and immunological differentiation into subclasses is important for patient outcomes. Differentiating lymphomas starts from the microscopic appearance continue with special stains and immunohistochemical analysis and in the end morphometric evaluation.

Marginal zone lymphoma incidence is 9.97/100,000 persons/year, but included all marginal zone lymphoma forms (nodal, non-nodal and splenic) $[1,2]$. Marginal zone lymphoma pathogenesis is unknown, and the cell of origin is only postulated 
considering that resemble monocytoid B cells. Nodal marginal zone lymphoma is a disease of adults that occurs in the sixth or seventh decade of life. Women are more affected than men, with a ratio of $2: 1$, and approximately $75 \%$ of patients present with localized lymphadenopathy.

\section{Material and methods}

To achieve morphometric examination were selected 6 of 8 cases identified as marginal zone lymphoma. Cases selected for morphometry were reviewed and representative regions were selected for study in each case. For each case analysis was done on five fields, for each field measuring the parameters of 20 cells, so morphometric measurements were made on 100 cells for each case. Were selected for measurement cell with clear nuclear outlines. The studied parameters were for cytoplasm: cytoplasmic area, maximum and minimum cytoplasmic diameter, cytoplasmic perimeter; for nucleus were measured: nuclear area, minimum and maximum nuclear diameter, nuclear perimeter, nuclear contour index, nuclear ellipticity index, nuclear irregularity index. Also the nucleo-cytoplasmic ratio was calculated in all studied cases. Nuclear indices were calculated from the measured nuclear area and perimeter. Elevated nuclear contour index shows the deviation from a circle, the other two indices, the ellipticity and irregularity, provides detailed data on the shape of the nucleus. Increasing irregularity index values above 3.54 reflect the existence of the more irregular nuclei as the index value is greater.

Getting morphometric data by measuring cellular parameters mentioned above was made using a Nikon E600 microscope equipped with Nikon DN100 camera for viewing microscopic slides chosen for evaluation, LuciaNet software running on a computer with Pentium processor and Windows operating system to obtain values parameters. Data obtained from the measurements were processed by Microsoft Excel calculating the average and standard deviation for each case.

\section{Results and discussion}

From international currently accepted data, marginal zone lymphoma replaces normal nodal architecture. In some cases, cancer cells can colonize reactive follicles, mimicking a follicular lymphoma. From the cytological point of view neoplastic cells are monocytoid, centrocit-like or they may mimic small round lymphocytes, and there may be some plasma cell differentiation that accompany these options [3,4]. Reactive follicles are usually identified in lymph nodes affected by marginal zone lymphoma.

From a microscopic point of view, in cases evaluated in the study, histological structure has been varied, the most common issue is the large or small lymphocyte cell proliferation with cleaved and uncleaved nuclei, the cleaved nuclei with irregular contour and uncleaved cells with round nucleus (Figures 1, 2, 3).

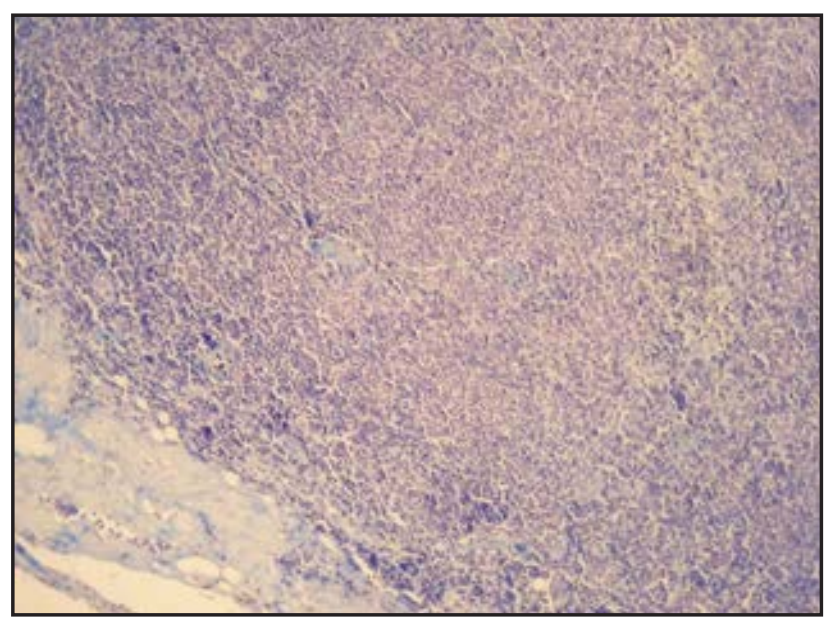

Figure 1 - Microscopic appearance of marginal zone lymphoma (Masson x 10)

This microscopic aspect is reflected in the values determined by morphometry. Nuclear irregularity index has values distributed over a close 
range between 3.85 and 4.01 with a mean value of $3.94 \pm 0.02$.

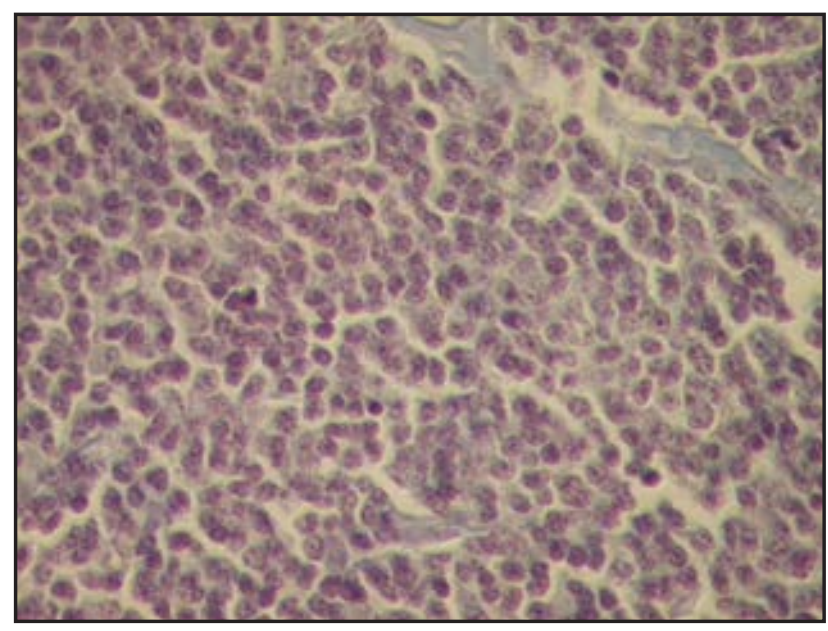

Figure 2 - Microscopic appearance of marginal zone lymphoma (Masson x 40)

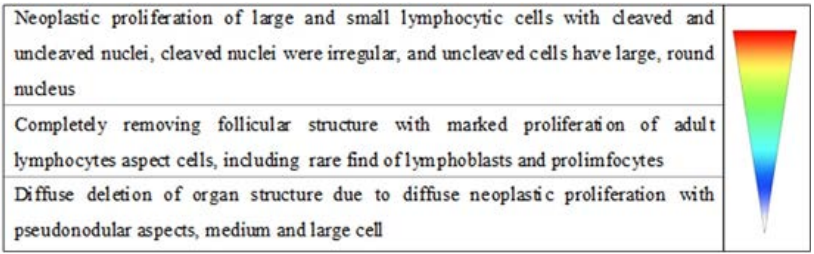

Figure 3 - Microscopic aspects in marginal zone lymphoma

Cytoplasmic area in marginal zone lymphoma cases varied within a relatively wide range of values between $50.52 \mu$ and $28.1 \mu$, average cytoplasmic areas in each study case highlighting the trend (they varied in a wide range of values between $33.39 \mu$ and $41.57 \mu$ ) (Table I). The standard deviation of the cytoplasmatic area calculated in each studied case has shown high and various values in a wide range, in line to the values obtained for the morphometric measurements of cytoplasmic areas. Standard deviation values were in the range from 5.42 to 8.95 (Figure 4). These high values of standard deviation and wider values range of cytoplasmic area. characterizes a cell population composed of large and small cells described in marginal zone lymphoma composition of studied cases.

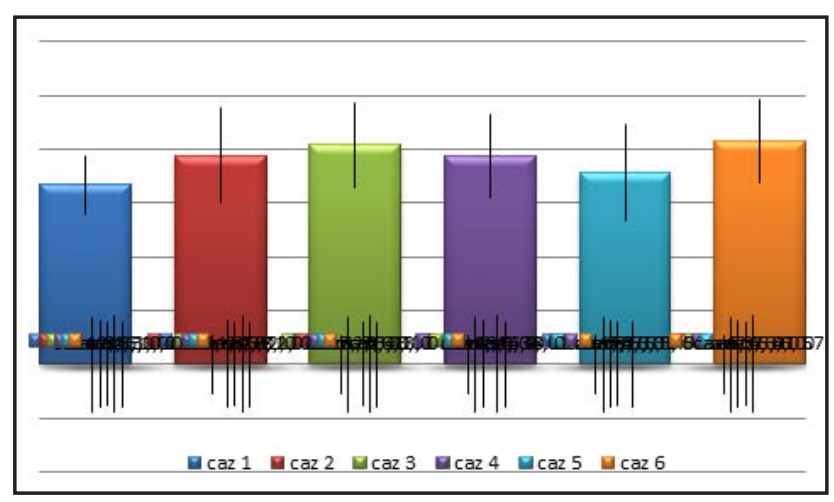

Figure 4 - Mean cytoplasmic area in marginal zone lymphoma cases

In international studies, large cell lymphomas have cytoplasmic areas with values above $51 \mu$ and small cell lymphomas have values below $27 \mu$. Measurements cytoplasmic areas average in this study revealed values between $33-41 \mu$, framing cell population between the two classes described above and confirming, along with high values of standard deviation, microscopic observations [5]. Values obtained by morphometric measurements for

Table I - Averages morphometric parameters measured in marginal zone lymphoma cases

\begin{tabular}{|c|c|c|c|c|c|c|c|}
\hline & 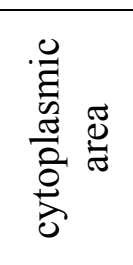 & 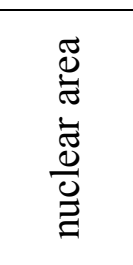 & 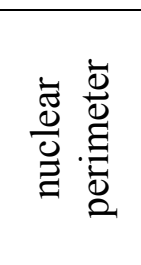 & 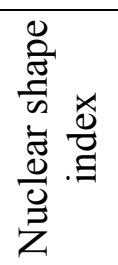 & 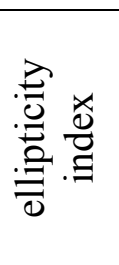 & 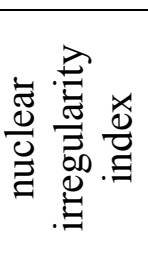 & 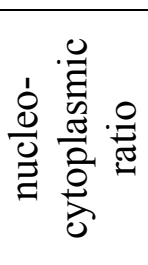 \\
\hline Overall average & 38.132 & 39.432 & 28.384 & 4.561 & 4.158 & 3.944 & 1.036 \\
\hline Overall SD & 7.731 & 7.637 & 1.530 & 0.253 & 0.241 & 0.028 & 0.010 \\
\hline The minimum & 28.11 & 29.51 & 25.27 & 4.09 & 3.81 & 3.85 & 1.011 \\
\hline The maximum & 50.52 & 51.63 & 31.32 & 5.11 & 4.67 & 4.01 & 1.053 \\
\hline
\end{tabular}


cytoplasmic areas shows that cell size is closer to the small cell lymphocytes so that it can be considered that small cells are the predominate component in studied cases.

Morphometric measurements of nuclear areas highlights the trend, bounding in the relatively wide range of values obtained, which characterized cytoplasmic areas also. So, nuclear area has values in the range of $29.51-51.63 \mu$ and the average nuclear areas value, is between $42.97 \mu$ and $34.79 \mu$. The standard deviation for the average nuclear areas meet the general trend of the measured parameter being within the range of 5.42 to 8.82 with an average of 7.63 but still lower than the standard deviation obtained for the cytoplasmic areas (Table I, Figure 5). This difference is due to the existence of cleaved and noncleaved cells in marginal zone lymphomas content. The larger nuclei nuclear areas, because of cleaved appearance, may have lower values approaching nuclear areas of small uncleaved nuclei.

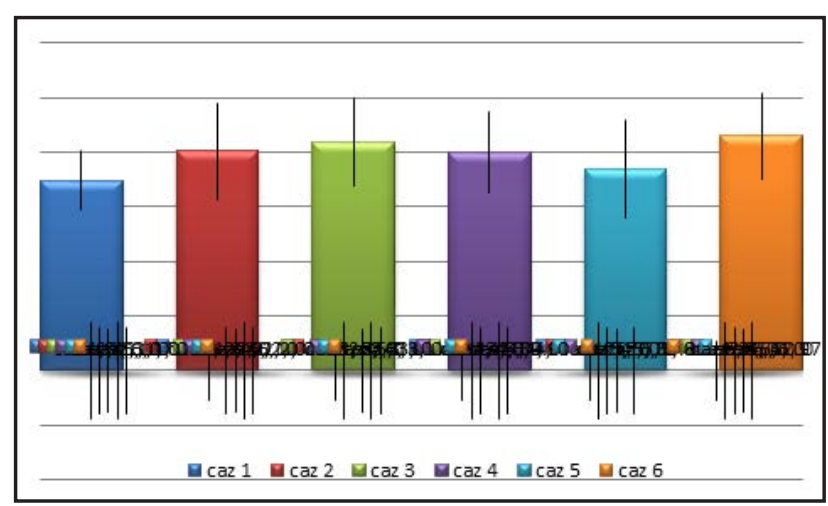

Figure 5 - The average nuclear area in marginal zone lymphoma cases

Nucleo-cytoplasmic ratio has a mean value of 1.036 and a standard deviation of 0.01 highlighting that the cells have relatively large nuclei and moderate amount of cytoplasm.

Morphometric nuclear contour index considers the degree of indentation of the nucleus being a parameter dependent on the perimeter and nuclear area. The higher nuclear contour index, the obvious nuclear indentations (have greater depth and lower angle). Nuclear contour index in this study, shows high values of marginal zone lymphoma cases, suggesting the presence of increased number of cleaved nuclei [6].

Ellipticity index is a parameter measured by morphometry who appreciate nucleus form depending on its axes, considering it to an ellipse, offers data closer to reality on nucleus shape and size, being in fact an improved version of the nuclear contour index , and can differentiate an irregular nucleus in the true sense, from a regular but elliptical nucleus. The higher nuclear ellipticity index values, the nucleus is more elongated [7]. Marginal zone lymphoma has a high value of ellipticity index (4.157), suggesting that cell nuclei in its composition are elliptical and may have or not indentations.

Nuclear irregularity index is calculated by adding a constant (3.54), which is the value of the ellipticity of a circle having a radius equal to unit, to substraction of nuclear contour index and the ellipticity index. In international literature nuclear irregularity indices values below 3.79 designate regular cell outline and values over 4.29 designate cleaved cells with irregular contour. Nuclear irregularity index is also an embodiment of the nuclear shape index much more accurate to characterize the shape of the nucleus $[7,8]$.

Nuclear irregularity index was shown to have small values in marginal zone lymphoma cases (3.943) suggesting a component rich in uncleaved cells and poorest cleaved cell nuclei. According to international literature marginal zone lymphoma reveals nuclear irregularity index values in the range of regular round or oval contour cells values, corresponding to higher nuclear contour index and ellipticity index values.

\section{Conclusions}

Marginal zone lymphoma morphometric parameters characterize a small cytoplasmic area (mean value of 38.132) and also a small nuclear area (mean value of 39.432), and small nucleocytoplasmatic ratio of about $1: 1$. Nuclear contour index is small, accompanied by a large ellipticity index and an small index of nuclear irregularity. Standard 
deviations for measured morphometric parameters are variable, having high values for cytoplasmic and nuclear area, highlighting the polymorphic nature of cells. Morphometric aspects, with corresponding microscopic aspects of large and small lymphocyte proliferation with cleaved and uncleaved nuclei, fit this form of lymphoma in terms of clinical outcome in indolent lymphomas category.
7. Pelstring, R.J. \& Swerdlow, S.H. (1987). Improved nuclear contour indices for lymphoid morphometry. Anal Quant Cytol Histol. 9(6), 469-74. PMID: 3325072

8. Swerdlow, S.H, Pelstring, R.H. \& Collins, R.D. (1990). Morphometric Analysis of Follicular CenterCellLymphomas. AmJournalofPathology. 134(4), 953-963. PMCID:PMC1877560

\section{References}

1. Morton, L.M., Wang, S.S. \& Devesa, S.S. et al. (2006). Lymphoma incidence patterns by WHO subtype in the United States, 1992-2001. Blood. 107, 265-276. PMCID: PMC1895348

2. Isaacson, P.G., Nathwani, B.N. \& Piris, M.A. et al. (2001). Nodal marginal zone B-cell lymphoma. In: Jaffe ES, Harris NL, Stein H, Vardiman JW et al. eds. World Health Organization classification of tumours. Pathology and genetics of tumours of haematopoietic and lymphoid tissues. (161). Lyon, France: IARC Press

3. Berger, F., Feldman, P. \& Thieblemont, C. et al. (2000). Non-MALT marginal zone B-cell lymphomas: a description of clinical presentation and outcome in 124 patients. Blood. 95, 19501956. PMID: 10706860

4. Camacho, F.I., Algara, P. \& Mollejo, M. et al. (2003). Nodal marginal zone lymphoma: a heterogeneous tumor. A comprehensive analysis of a series of 27 cases. Am J Surg Pathol. 762771. PMID: 12766579

5. Van Der Valk, P., Mosch,A., Kurver, P.J. \& Meijer, C.J.L.M. (1983). Morphometric characterization of 52 B cell non-Hodgkin's lymphomas. JClin Pathol. 36, 289-297. DOI: 10.1136

6. Tosi, P., Leoncini, L., Spina, D. \& Del Vecchio, M.T. (1984). Morphometric Nuclear Analysis of Lymphoid Cells in Center Cell Lymphomas and in Reactive Germinal Centers. Am Journal Pathol. 117(1), 12-17. PMID:6385724, PMCID:PMC1900559 